\title{
Improving the quality of kidney transplant recipient discharge summaries
}

\author{
Authors: Kerry Hall, Christopher Patrick Uy, Rupert Bright, Michelle Willicombe and Philip Webster
}

\section{Introduction}

Hammersmith Hospital transplants around 200 kidneys per year and follows up around 300 transplant patients per week in the outpatient department. Upon discharge from hospital, the immediate postoperative period as an outpatient is crucial as clinicians make key decisions regarding management in order to maximise transplant success. These decisions are tailored to a patient's individual factors such as donor type, donor age, histoincompatibility (human leukocyte antigen (HLA) mismatches) and ischaemia times. This information is obtained from many sources in the pre-, peri-, and postoperative periods - for example, from the organ retrieval team, from the on-call tissue typist, and from the transplant surgery team.

Following the transition to Cerner Powerchart, data is more accessible to the teams involved in managing the patient. However, the data can be fragmented across several different Cerner entries. Unless a succinct discharge summary is written, the key details can take time to search for in the outpatient setting. Feedback from the renal consultants suggests that information gathering at the first outpatient visit can significantly prolong the appointment. This can therefore slow down the busy transplant clinics and impact upon management decisions if any information is unavailable. Making all of this information readily available upon discharge streamlines the outpatient process and allows for timely and informed decisions to be made in clinic. The aim of this project was to improve kidney transplant discharge summary quality by improving the documentation of risk factors that impact transplant survival.

\section{Materials and methods \\ Using Plan, Do, Study, Act (PDSA) quality improvement methodology, the current guidelines for transplant recipient monitoring were reviewed, and with guidance from the renal consultant cohort, a discharge summary template was designed that included all pertinent risk factors impacting transplant survival. The inpatient journey of transplant recipients was analysed and key areas were identified where risk factors became available (Table 1). We also identified the key individuals required to implement the}

Authors: Imperial College Healthcare NHS Trust

\begin{tabular}{ll}
\hline Table 1. Clinical document risk factors \\
\hline $\begin{array}{ll}\text { Clinical document } \\
\text { Clinical clerking }\end{array}$ & $\begin{array}{l}\text { Risk factor } \\
\text { End stage renal disease (ESRD) cause, dry } \\
\text { weight, transfusion history }\end{array}$ \\
Organ offer notes & $\begin{array}{l}\text { Type of transplant, donor age, donor } \\
\text { creatinine }\end{array}$ \\
Tissue typing report & $\begin{array}{l}\text { HLA mismatch, presence of donor specific } \\
\text { antibody }\end{array}$ \\
Operating notes & $\begin{array}{l}\text { Date of transplant, transplant anatomy, } \\
\text { cold ischaemic time, warm ischaemic time }\end{array}$ \\
Drug chart & $\begin{array}{l}\text { Immunosuppression induction and } \\
\text { maintenance }\end{array}$ \\
Blood results & Discharge creatinine
\end{tabular}

template successfully, and specifically targeted them via various communication strategies when introducing it. The discharge summary template was launched to the renal team in January 2019 and served as a self-audit tool for a complete summary. To assess the change, data was collected retrospectively from the discharge summaries from August 2018 to October 2018 and compared with those written in January 2019 to February 2019. The discharge summaries were tallied for the presence of each risk factor.

\section{Results}

The presence of discharge summary risk factors increased by an average of $43.81 \%$ overall after the launch of the template. Prior to the template, the most commonly omitted risk factors were donor creatinine $(83.33 \%)$, recipient dry weight $(87.50 \%)$, and blood transfusion history (91.77\%). The most improved risk factors were immunosuppression induction agent (increase of $72.92 \%$ ), donor creatinine (increase of $69.04 \%$ ), and delayed graft function (increase of $68.75 \%$; Fig 1 ).

\section{Conclusion}

PDSA methodology can improve the quality of transplant discharge summaries to include important risk factors. The success of this project has improved the availability of 


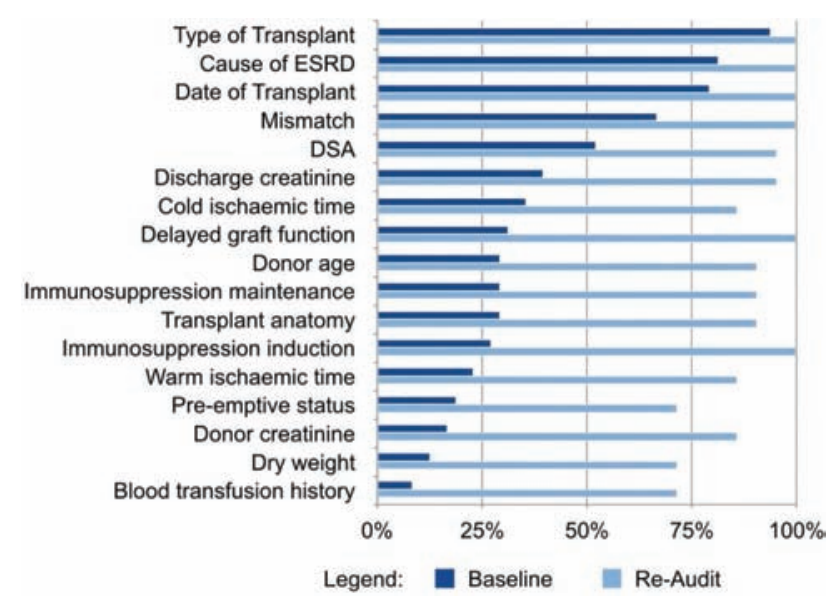

Fig 1. Risk Factor Percentage information for transplant recipients' risk factors to all clinicians in the renal team.

\section{References}

1 ACT Academy. Plan, Do, Study, Act (PDSA) cycles and the model for improvement. ACT Academy, 2018. Available at: https:// improvement.nhs.uk/documents/2142/plan-do-study-act.pdf [Accessed 26 February 2019].

2 Baker R, Mark P, Patel R, Stevens K and Palmer N. Renal association clinical practice guideline in post-operative care in the kidney transplant recipient. BMC Nephrology 2017;18:174.

3 Kidney Disease: Improving Global Outcomes (KDIGO) Transplant Work Group. KDIGO clinical practice guideline for the care of kidney transplant recipients. Am J Transplan 2009;9:S1-157. 